\title{
A98-37313
}

AIAA $-98-4357$

\section{Mathematical Modeling AND ExPeRIMEntal IDENTIFICATION OF A MODEL HELICOPTER}

\author{
S. K. Kim* and D. M. Tilbury ${ }^{\dagger}$ \\ Department of Mechanical Engineering and Applied Mechanics \\ University of Michigan, Ann Arbor, MI 48109-2125 \\ sungk@engin.umich.edu, tilbury@umich.edu
}

\begin{abstract}
This paper presents a new mathematical model for a model-scale helicopter. Working from first principles and basic aerodynamics, the equations of motion for full six degree-of-freedom motion are derived. The control inputs considered are the four pilot commands from the radio transmitter: roll, pitch, yaw, and thrust. The model helicopter has a fast time-domain response due to its small size, and is inherently unstable. A flybar is used to augment the stability of a model helicopter to make it easier for a pilot to fly. The main contribution of this paper is to model the interaction between the flybar and the main rotor blade; it is shown how the flapping of the flybar increases the stability of the model helicopter as well as assists in its actuation. After the mathematical model is derived, some preliminary system identification experiments and results are presented. The paper ends with conclusions and a short description of future work.
\end{abstract}

\section{Introduction}

$\mathbf{I}$ $T$ has been more than 20 years since the first commercial model helicopter was conceived, and since then, the design has significantly improved. Model helicopters are now well within the reach of many hobbyists and are also often used for commercial purposes, such as crop dusting or sport-event broadcasting. However, model helicopters are inherently unstable. Even with improved stability augmentation devices, a skilled, experienced pilot is required to control them during flight.

As a small, dynamically fast, unstable system, a model helicopter makes an excellent testbed for nonlinear control experiments. As a highly maneuverable machine, it also is an excellent testbed for path planning algorithms for autonomous robots. The integration of nonlinear control and path planning is our main interest in this project. As a preliminary step, this paper describes the new mathematical model that we have derived for a model helicopter control system, as well as some preliminary system identification experiments we have conducted. The outline of the paper is as follows. First, we briefly review some previous work on helicopter modeling and control. We then describe the new mathematical

\footnotetext{
${ }^{*}$ Ph.D. Student, University of Michigan, AIAA Student Member

$t^{\dagger}$ Assistant Professor, University of Michigan

Copyright $(1998$ by Sung K. Kim. Published by the American Institute of Aeronautics and Astronautics, Inc. with permission.
}

model that we have derived, working from first principles and basic aerodynamics. We then describe the system identification algorithm that we have used and present our preliminary results. We end with conclusions and a description of future work.

\section{Previous Work}

In the past several years, there have been a number of researchers interested in model helicopter control, and they have had various degree of success.

\section{System Identification}

A group at Caltech attempted to capture the main dynamic features of a model helicopter near hover with a linear time-invariant model. A MIMO identification algorithm was used to account for the significant cross-couplings in the model helicopter. Bendotti et al. ${ }^{1}$ set up a model helicopter on a stand to provide three degrees of freedom: pitch, roll, and yaw rotations. Translational movements such as $x$, $y$, and $z$ positions were neglected. The identification was carried out using a quadratic weighing factor with an iterative Gauss-Newton algorithm. The match between simulation and experiment was good in the pitch and roll directions, but was poor in the yaw direction due to the actuation asymmetry. Controllers were designed using $H_{\infty}$ and LQG methods and the linear model that had been identified. Both controllers showed good response in disturbance rejection and command tracking; the $H_{\infty}$ controller 
had a faster response.

Another group from Caltech ${ }^{2}$ did a similar identification and designed a LQR controller. The realtime control and data acquisition ran on the PC, which hosted the pulsewidth modulated IO board and Polhemus sensor board. ${ }^{3}$ Again, the controller showed good performance only for pitch and roll response. Yaw response was poor for the same reason as above. Significant improvement on the yaw response was achieved when a separate loop shaping controller based on a lead-lag design was implemented.

\section{Fuzzy Control}

Dr. Sugeno from the Tokyo Institute of Technology ${ }^{4}$ has had a considerable amount of success in flying the model helicopter for commercial purposes. The project's goal was to develop a controller for an unmanned helicopter that can operate under hostile conditions. The control system was designed using fuzzy control theory. The integrated control system, ranging from low level basic flight modes to high level supervisory control, takes a human voice as its input. Because human language voice commands are naturally imprecise or 'fuzzy,' the fuzzy logic framework was a good fit. The primary issue here was to design a controller that can easily include qualitative information as well as quantitative information. Some pre-experiment simulation was done on a Silicon Graphics IRIS workstation and a PC. The fuzzy controller rules were first constructed with the help of a actual human pilot's experience and knowledge. The rules were then tested and reformulated using the simulator. The helicopter was equipped with various sensors such as camera, gyroscope for $3 \mathrm{D}$ acceleration, Doppler speedometer, magnetic compass, laser altimeter, and GPS.

\section{Unmanned Aerial Vehicle Competition}

The annual unmanned aerial vehicle competition has been organizing a number of universities to complete a task of recognizing and moving an object to a designated target. So far, the competitors have typically been concentrating on the sensory issues such as the image processing, with a lesser emphasis on the control problems. Among the participating universities, USC used a concept called "behavior based control", 5,6 which implements a number of complex tasks with a collection of simple, interacting behaviors in parallel. Their approach seeks to use a behavioral based approach as a structure to unify navigation, motor control, and vision. This approach was inspired by the distributed yet unified control of biological systems. To maintain the safety of the helicopter, the lower-level behaviors may 'negotiate' with a mid-level to satisfy both sets of behavioral criteria. They claim this approach was useful in building an integrated control system; an open issue is the determination of a set of rules and principles for creating behaviors. The MIT, Boston University and Draper Lab team ${ }^{7}$ was successful in building an autonomous model helicopter designed to hover, fly around, and recognize five randomly placed drums during the 1996 International Aerial Robotics Competition. The system consisted of the helicopter with various sensors such as GPS, IMU, altimeter, and compass, as well as a ground control station, a vision processor, and a safety pilot. The control system is divided into four closed loops.for roll, pitch, yaw, and collective/throttle with integrators to eliminate steady state errors. Pre-determined trim positions were used on those loops. They used relatively simple control laws to minimize the development time and be flexible to the changes in helicopter configuration. Again, their research effort was concentrated more on the sensor issues and the interactions between the various components than on the control structure itself.

\section{Other UAV}

The Naval Research $\mathrm{Lab}^{8}$ has designed an airplane style UAV which is intended to be a missile decoy. It has an electric motor for a nose mounted propeller, three fiber-optic rate gyro sensors for the pitch, roll, and yaw axes, a barometric altimeter, and a pitot-static airspeed sensor. All the actuator dynamics are based on linear dynamic equations, and the single-axis control law is used for attitude and altitude control. From the launch through the cruise transition phase, the trajectory is based on a first-order linear fit to find the desired pitch angle profile based on 6DOF simulation. The reference pitch position is time-scheduled to follow the nominal trajectory. Various parameters are based on a wind tunnel experiment. Although the paper did not address the issues regarding how the aircraft would follow the pre-planned trajectory once it reached the cruise phase in satisfying the specific mission goal, it demonstrated the potential capability of the craft as a missile decoy.

In terms of UAV control, Kaminer et al. ${ }^{9}$ pointed out that under a shifting wind disturbance, the traditional control scheme, in which the guidance and control parts are separate, may be inadequate to satisfy the precise tracking and frequent heading change necessary. To solve this problem, they examined a guidance and control scheme where the two are combined, so that the guidance law becomes an integral part of the feedback control system. With 
such design specifications as zero steady state error and certain bandwidth requirements, they devised a state-space coordinate system in which the linearization of the plant along a reference trajectory is time-invariant. This realization results in trajectories that consist of straight lines, arcs of circles of constant radii, and any combinations thereof. The control system itself is an LQR design. Their simulation showed a good result for an aircraft following a descending helical trajectory. However, the scheme only applies to certain specific trajectories as mentioned above, so it remains uncertain how useful the technique could be to a real system.

Sikorsky Aircraft ${ }^{10}$ has been developing a saucertype VTOL UAV called Cypher to meet various civil and military requirements. It has two counterrotating, $4 \mathrm{ft}$ long, coaxial, four-bladed main rotors shrouded by the $6.5 \mathrm{ft}$ diameter main frame. Similar to a helicopter, it uses collective and cyclic pitch control for movement, powered by a $60 \mathrm{hp}$ rotary engine. Sensors include radar, gyros, accelerometer, GPS, video camera, etc. To achieve a simple operator/vehicle interface, the operator is only required to send out basic maneuver commands such as takeoff, hover, cruise, desired heading, etc. Linear state space models are used to develop control laws and to determine specifications for servo and sensor bandwidths. The vehicle successfully demonstrated reconnaissance and surveillance capability, and is being improved to accomplish such tasks as mine-deploying and scouting missions. The vehicle is not yet completely autonomous nor is it capable of following a pre-determined trajectory.

\section{Other research}

At Purdue, ${ }^{11}$ a student derived the dynamic equation of a model helicopter's vertical motion using blade element theory. For the experiment, a model helicopter was affixed on a stand to let it move only vertically. Uncertain parameters were estimated by interpolating the results from number of experiments with different parameters. After linearizing the dynamic model around the hover condition, controllers were designed using full state feedback poleplacement, LQR, and neural network techniques. All the controllers showed satisfactory performance.

Furuta et al. ${ }^{12}$ derived a mathematical model of a model helicopter fixed on a stand, free to rotate around the pitch, roll, and yaw axis. Conservation of angular momentum was used to obtain the model. Realizing that the model helicopter is hard to control manually, they designed a stable tracking controller using the state space method. This stabilizing control input is then used to compare and validate the

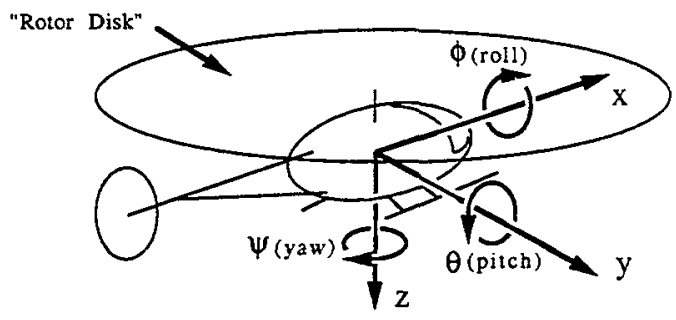

Fig. 1 The coordinates defined. The orientation variables, roll, pitch, yaw $(\phi, \theta, \psi)$ and the position variables $(x, y, z)$ are relative to the body frame (fixed to the helicopter).

derived mathematical model with the experimental result. They realized that to be able to control the model helicopter in a full 6DOF situation, they need to expand the model to other degrees of freedom including the vertical motion. The model they derived was based on the full-scale helicopter modeling because they modeled a flapping rotor hub with spring, without explicitly taking the flybar into account.

Azuma $^{13}$ from University of Tokyo derived a mathematical model of a rigid rotor system. In this rotor system, each rotor blade is hinged but is spring loaded. He carefully derived a equation of motion using complex variables considering how different flapping stiffness affects the response of the system. Since this model does not take the dynamics of the helicopter as a whole, the validity of the model has not been fully proven.

\section{Mathematical model}

In this section, we will derive the dynamic equations of motion for the model helicopter including its actuator dynamics. We will use results from rigid body dynamics, ${ }^{15}$ as well as basic aerodynamics and helicopter theory. ${ }^{14,16}$

\section{Model vs. full scale helicopters}

Before deriving the model helicopter dynamics, it is important to consider the differences between a full-scale helicopter and a model helicopter. First of all, a model helicopter has a much faster timedomain response due to its small size. Therefore, without employing an extra stability augmentation device, it would be extremely difficult for a human pilot to control it. A large control gyro with an airfoil, often referred to as a flybar, is almost always used nowadays to improve the stability characteristic around the pitch and roll axes and to minimize 
the actuator force required. Also, the tail rotor control for the model helicopter is assisted by an electronic gyro to further stabilize the yaw axis. Most full scale helicopters do not have such a control gyro on the rotor system. The large inertia of the rotor and fuselage and the flapping rotor hinge provide adequate stability.

Secondly, most model helicopters do not have a flapping hinge on the rotor to maximize the control power. Full scale helicopters often use either a free flapping rotor hinge or a spring-mounted hinge; these are usually absent on a model helicopter.

\section{Rigid Body Equations}

We will model the helicopter as a rigid body moving in space. As shown in Figure 1, we use the variables $(x, y, z)$ to represent the position of the helicopter in body coordinates. We use the variables $(\phi, \theta, \psi)$ to represent the roll, pitch, and yaw angles of the helicopter with respect to the body coordinates. Because it is a rigid body, the helicopter's position and orientation in body coordinates will always be zero; however, the velocity and acceleration expressions are greatly simplified by using these coordinates. We will assume the rotor system is completely rigid (there is no aeroelasticity effect), and that the the airfoil is symmetric and non-twisted. The aerodynamic interaction between the rotor and the fuselage is neglected. The aerodynamic expressions are based on 2-D analyses. This type of modeling is often called level-1 modeling and is appropriate for low bandwidth control and to observe the parametric trends for flying qualities and performance studies. ${ }^{14}$

The standard rigid body dynamical equation will be used to model the motion of the helicopter in its environment. The state vector $q=(x, y, z, \phi, \theta, \psi)^{T}$ contains the position and orientation information for the helicopter. Expressing the equation in coordinates gives ${ }^{15}$

$$
\begin{gathered}
{\left[\begin{array}{cc}
m I_{3 \times 3} & 0 \\
0 & I
\end{array}\right] \ddot{q}+R_{I B}^{T} \dot{R}_{I B}\left[\begin{array}{cc}
m I_{3 \times 3} & 0 \\
0 & I
\end{array}\right] \dot{q}=} \\
{\left[\left(\begin{array}{c}
-D_{F_{x}} \\
-D_{F_{y}} \\
T-D_{F_{z}}
\end{array}\right)+R_{I B}^{T}\left(\begin{array}{c}
0 \\
0 \\
m g \\
M_{\phi} \\
M_{\theta}-T \ell_{r} \\
M_{\psi}+T_{m}-I_{r} \dot{\Omega}-K_{g} \dot{\psi}
\end{array}\right]\right.}
\end{gathered}
$$

The rotation matrix $R_{I B}^{T}$ transforming the inertial coordinates into body coordinates using yaw-pitchroll $(Z Y X)$ Euler angles is given by

$R_{I B}=e^{(\hat{z} \times) \psi} e^{(\hat{y} \times) \theta} e^{(\hat{x} \times) \phi}$

$$
\begin{aligned}
= & {\left[\begin{array}{ccc}
\cos \psi & -\sin \psi & 0 \\
\sin \psi & \cos \psi & 0 \\
0 & 0 & 1
\end{array}\right] \cdot\left[\begin{array}{ccc}
\cos \theta & 0 & \sin \theta \\
0 & 1 & 0 \\
-\sin \theta & 0 & \cos \theta
\end{array}\right] . } \\
& {\left[\begin{array}{ccc}
1 & 0 & 0 \\
0 & \cos \phi & -\sin \phi \\
0 & \sin \phi & \cos \phi
\end{array}\right] }
\end{aligned}
$$

The cross " $x$ " notation is used to represent the skewsymmetric cross-product matrix. For a vector $a=$ $\left[\begin{array}{lll}a_{1} & a_{2} & a_{3}\end{array}\right]^{T}$,

$$
a \times=\left[\begin{array}{ccc}
0 & -a_{3} & a_{2} \\
a_{3} & 0 & -a_{1} \\
-a_{2} & a_{1} & 0
\end{array}\right]
$$

The rotational inertia matrix of the helicopter is given by

$$
I=\left(\begin{array}{ccc}
I_{x x} & 0 & -I_{x z} \\
0 & I_{y y} & 0 \\
-I_{x z} & 0 & I_{z z}
\end{array}\right)
$$

The terms on the right-hand side of the rigid body equation (1) include both the applied forces and disturbances. The $D$ terms represent drag forces; these will be treated as disturbances in our model. The mass of the helicopter is given by $m$, and the fuselage inertias are $I_{x x}, I_{y y}, I_{z z}$. Terms such as $I_{x y}$ and $I_{y z}$ are zero due to the symmetry of the helicopter with respect to the $x-z$ plane. Although $I_{x z}$ is nonzero, because the helicopter is not symmetric with respect to the $x-y$ plane, it is typically much smaller than the other terms. We have included it in the model for completeness. The rotor rotational inertia is $I_{r}$. The rotor angular velocity is $\Omega$, and the offset between the rotor axis and the helicopter's center of gravity is $\ell_{r}$. Usually this offset is expected to be zero for better handling quality. Nevertheless, we will assume this quantity is non-zero for generality. The gravitational acceleration constant is $g$. It is assumed that the helicopter's center of gravity is in-line with the rotor axis laterally.

The four independent inputs are $T$, the net thrust generated by the rotor, and $M_{\phi}, M_{\theta}, M_{\psi}$, the net moments acting on the helicopter, which are applied by a pilot. The mechanisms for creating these inputs will be described in the following section. The torque applied by the motor, $T_{m}$, is related to the thrust $T$ and cannot be controlled independently. The electronic gyro acts as a damper on the yaw motion; we will use a simple linear model, $K_{g} \dot{\psi}$ for this gyro, although more sophisticated (PI controlled) gyros have recently become available.

\section{Model helicopter actuation}

A model helicopter moves forward when a pitching moment is first applied and the fuselage is tilted 


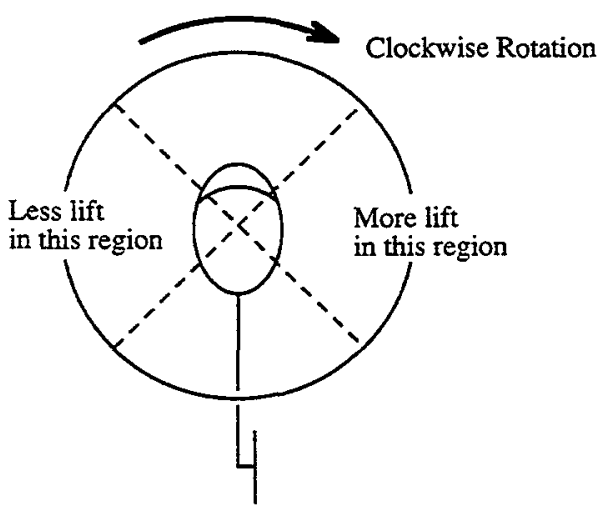

Fig. 2 The top view of the helicopter. The lift distribution on the rotor disk when a forward cyclic (pitch forward) input is applied. The precession effect will pitch the helicopter forward.

forward. The thrust vector $T$ then gives a forward component for a forward thrust. A full scale helicopter only requires a forward tilt of the rotor disk to move forward while the fuselage stays level, ${ }^{16}$ but this type of maneuver is not possible with a model helicopter.

There are four inputs available to the pilot of a model helicopter. These are physically controlled by two joysticks on the radio transmitter, each with two degrees of freedom. The left joystick commands throttle with collective pitch (up/down) and yaw (left/right), and the right joystick commands pitch cyclic (up/down) and roll cyclic (left/right). This is the most popular configuration used in the U.S. (type $\mathrm{II}^{*}$ ). The four values representing the positions of the sticks are encoded in a pulse-width modulated (PWM) signal, and sent via radio link to the helicopter. We will use these four positions $\left(\delta_{t}, \delta_{\phi}, \delta_{\theta}, \delta_{\psi}\right)$ as the inputs to our actuator dynamic equations because of the way we will control the helicopter later.

The throttle command $\left(\delta_{t}\right)$ controls the power to the main motor $\left(T_{m}\right)$ as well as the collective pitch $\left(\theta_{0}\right)$ of the rotor blades. As the blade pitch increases, more lift is created, and the rotational motion of the main rotor blade is converted into vertical thrust. Usually, the relationship between $\theta_{0}$ and $\delta_{t}$ is linear $\left(\theta_{0}=K \delta_{t}\right.$, where $K$ is some constant). Meanwhile, an adequate torque $T_{m}$ is applied to keep $\Omega$ constant. Sometimes, an electronic throttle governor is used for this purpose, but mostly a fixed simple smooth curve determining the necessary functional relationship between $T_{m}$ and $\delta_{t}$ is programmed into the radio transmitter. This curve is traditionally obtained via trial and error. The yaw command $\left(\delta_{\psi}\right)$

\footnotetext{
*In some countries, people prefer the yaw and the roll controls switched (type I).
}

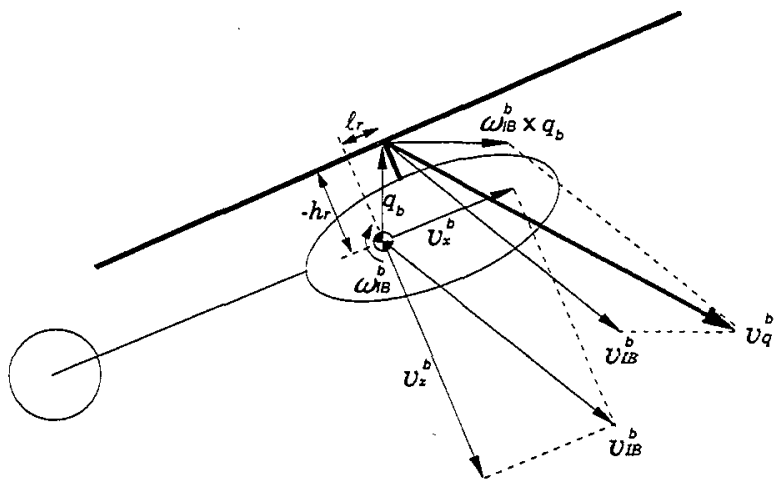

Fig. 3 Vector diagram explaining how the velocity $v_{q}^{b}$ of the hub point $q_{b}$ is calculated. The linear velocity of the helicopter CG is $v_{I B}^{b}$ and its angular velocity is $\omega_{I B}^{b}$. All velocities are measured in body coordinates. For clarity in the figure, lateral motions are neglected.

controls the pitch of the tail rotor blade. The tail rotor on a helicopter is used to counteract the yaw moment created by the main rotor blade; thus, altering the amount of pitch on the tail rotor can create more or less total yaw moment for the helicopter. The pitch and roll commands influence the cyclic control, varying the cyclic pitch $\left(\theta_{c y c}\right)$ of the rotor blades around each cycle of rotation, creating different amounts of lift in different regions (as shown in Figure 2). These differing amounts of thrust create a moment around the rotor hub, and can thus create pitch and roll moments on the helicopter.

Before developing the dynamic equations, we introduce some basic aerodynamic terms that will be required. The advance ratio, $\mu$, and the descent ratio, $\nu$, represent the airspeed components parallel to and perpendicular to the rotor disk respectively. ${ }^{17}$ They are close to zero when the helicopter is hovering. Both quantities are non-dimensionalized by $R \Omega .^{\dagger}$ To define these quantities, we first need to find the velocity of the hub point with respect to the inertial coordinate frame represented in body coordinates. We denote this velocity by $v_{q}^{b}$. The angular velocity of the body frame as viewed in the body frame is $\omega_{I B}^{b}$. The velocity of the $C G$ relative to the inertial frame is $v_{I B}^{b}$. The coordinate of the rotor hub point is $q_{b}=\left[\begin{array}{lll}\ell_{r} & 0 & -h_{r}\end{array}\right]^{T}$. The constants $-h_{r}$ and $\ell_{r}$ are the offsets of the rotor from the helicopter's center of gravity in the $x$ and $z$ directions respectively; the rotor hub is in-line with

\footnotetext{
'Strictly speaking, $R$ is the rotor span, excluding the rotor hub length. However, since the model helicopter's rotor blade remains nearly rigid due to its short length ( 0.4 to 0.8 meter), high rotor speed (1300 to $1900 \mathrm{rpm}$ ), and hingeless hub design, $R$ is assumed to be the distance between the rotor axis and the rotor tip.
} 
the CG in the $y$ direction. The geometry is sketched in Figure 3.

$$
\begin{aligned}
v_{q}^{b} & =\omega_{I B}^{b} \times q_{b}+v_{I B}^{b} \\
& =R_{I B}^{T} \dot{R}_{I B}\left[\begin{array}{c}
\ell_{r} \\
0 \\
-h_{r}
\end{array}\right]+R_{I B}^{T}\left[\begin{array}{c}
\dot{x} \\
\dot{y} \\
\dot{z}
\end{array}\right]_{I}
\end{aligned}
$$

where,

$$
R_{I B}^{T} \dot{R}_{I B}=\left[\begin{array}{c}
\dot{\phi}-\dot{\psi} \sin \theta \\
\dot{\theta} \cos \phi+\dot{\psi} \cos \phi \sin \theta \\
-\dot{\theta} \sin \phi+\dot{\psi} \cos \phi \cos \theta
\end{array}\right] \times
$$

The advance ratio, $\mu$, which is the airspeed component parallel to the rotor disk, is the magnitude of the first two elements of $v_{q}^{b}$, and the descent ratio, $\nu$, which is the airspeed component perpendicular to the rotor disk, is the magnitude of the third element of $v_{q}^{b}$.

$$
\begin{aligned}
\mu & =\frac{1}{R \Omega} \sqrt{\left(v_{q 1}^{b}\right)^{2}+\left(v_{q 2}^{b}\right)^{2}} \\
\nu & =\frac{1}{R \Omega}\left|v_{q 3}^{b}\right|
\end{aligned}
$$

The rotor solidity, $\sigma$, is the ratio between the rotor blade area and the rotor disk area. It indicates how "solid" the rotor disk is ${ }^{16}$ and is taken to be the number of blades (2) times the area of a rotor blade $(2 c R)$ divided by the area defined by the rotor disk $\left(\pi R^{2}\right)$.

$$
\sigma=\frac{2 c}{\pi R}
$$

The inflow ratio, $\lambda$, is the net value of the descent ratio $\nu$ and the induced air velocity, the velocity of the air through the rotor blade, $v_{i}$. It is nondimensionalized by $R \Omega$.

$$
\lambda=-\nu+\frac{v_{i}}{R \Omega}
$$

The lift curve slope, $a$, is the slope of the function of the lift vs. angle of attack of the main rotor blade.

\section{Flybar dynamics}

The dynamics of the rotor and the flybar are the most significant nonlinearities involved in the creation of the forces and moments on the helicopter. The actuator dynamics include as states the flapping angle and velocity $(\beta, \dot{\beta})$ of the flybar and the position and angular velocity $(\xi, \Omega)$ of the main rotor blade. As mentioned before, the flybar plays a major role in augmenting the stability of the helicopter. This system is often called as a Bell-Hiller mixer, because it takes advantage of two different cyclic control systems, as shown in Figure 5. Cyclic control is the mechanism by which the rotor blade's pitch is changed in a rotation so that an unequal distribution of the lift applies a moment around the rotor hub. This moment then provides pitch and roll attitude control as depicted in Figure 2. The Bellmixer allows the blade pitch to be changed directly from the cyclic servo actuator. It is fast in response, but lacks stability. Meanwhile, the Hiller-mixer allows the pitch of the flybar to be changed instead of the pitch of the blade. The flybar then flaps, and this flapping motion causes the pitch of the main blade to change.

There is a direct relationship between the cyclic input applied to the main blades $\delta_{c y c}$ (which is the function of stick commands $\delta_{\theta}$ and $\delta_{\phi}$ ) and the cyclic angle of the rotor blades $\theta_{c y c}$. A similar relationship exists between the cyclic input applied to the flybar $\delta_{f l y}$ and the flapping angle of the flybar $\beta$. The orientation of the main blade is given by $\xi$. Note the $90^{\circ}$ phase difference between $\delta_{c y c}$ and $\delta_{f l y}$, due to the geometry of the rotor/flybar assembly sketched in Figure 5.

$$
\begin{aligned}
& \delta_{c y c}(\xi)=-\delta_{\theta} \sin \xi-\delta_{\phi} \cos \xi \\
& \delta_{f l y}(\xi)=\delta_{\theta} \cos \xi-\delta_{\phi} \sin \xi
\end{aligned}
$$

An important assumption at this point is that the rotor system does not apply reaction forces back to the actuators, including the flybar (the flybar is considered to be another actuator to the main blades). This is equivalent to assuming the actuators are able to apply infinite amount of forces to the airfoils. We also neglect the influence of $\mu$ and $\nu$ on the flybar due to its relatively small wing surface.

When a cyclic input is applied by the pilot, the flybar creates lift which tilts the flybar disk. The flybar acts not only as a main blade angle actuator but also as a stabilizer. If the cyclic input were applied to the main blades only, large control forces on the cyclic servo actuators would be required. ${ }^{18} \mathrm{By}$ applying the cyclic control to the flybar and allowing the flybar to apply a secondary cyclic input to the main blade, the servo load is significantly reduced.

The flybar is hinged freely on the main axis and rotates around the main axis. Its angle, $\beta$, is measured with respect to the plane perpendicular to the main rotor axis. The rotation matrix which relates the flybar position to the body coordinates of the helicopter is denoted $R_{B F}$; as before, the rotation matrix between the helicopter body frame and the inertial frame is $R_{I B}$. The rotation matrix relating the flybar frame to the inertial frame is thus the product of the two: $R_{I F}=R_{I B} R_{B F}$.

The pitch angle of the flybar in the helicopter body coordinates is $\beta$; its yaw angle is $\xi+90^{\circ}$. The 
rotational inertia of the flybar $\widehat{I}$ is unified as $I_{f}$; this is a reasonable assumption because most of the flybar mass is concentrated at the tip region. The angular velocity of the flybar $\omega_{F}$ involves the velocity of the flybar and the main axis simultaneously; it can be found by computing $R_{I F}^{T} \dot{R}_{I F}$ and extracting terms from the elements in $R_{I F} \cdot{ }^{15}$

$$
\begin{aligned}
I_{f} & =\int_{R_{1}}^{R_{2}} r^{2} m_{F} d r \\
\hat{I} & =\operatorname{diag}\left[\begin{array}{lll}
0 & I_{f} & I_{f}
\end{array}\right] \\
\left(\omega_{F} \times\right) & =R_{I F}^{T} \dot{R}_{I F}
\end{aligned}
$$

The external moment applied on the flybar around the pitch axis is $\tau_{F}$, which is the aerodynamic lift term. To find the total torque, we integrate along the length of the flybar. Since we will only be interested in the second of these three equations, we will not consider the first and the third elements in any detail.

$$
\tau_{F}=\left[\begin{array}{lll}
\tau_{F_{1}} & \int_{R_{1}}^{R_{2}} r d L & \tau_{F_{3}}
\end{array}\right]^{T}
$$

The lift element $d L$ depends on the angular velocity of the flybar and its angle of attack $\theta_{A O A}$. The angle of attack of the flybar will be influenced by the pilot input $\delta_{f l y}$ and the second element of the angular velocity vector of the flybar, denoted $\omega_{F_{2}}$.

$$
\begin{aligned}
d L & =\frac{1}{2} \rho(\Omega r)^{2} a \theta_{A O A} c d r \\
\theta_{A O A} & =-\frac{\delta_{f l y}}{L_{5}}-\frac{\omega_{F_{2}}}{\Omega}
\end{aligned}
$$

The roll motion of the flybar only affects the lift created by changing the angle of attack. Because this lift term is already included into $\tau_{F}$, the roll motion of the flybar is neglected. The yaw motion of the flybar follows the angle of the main rotor blade. Once the external force, angular velocity, and inertia have been defined, the motion of the flybar can be described using the Euler equation.

$$
\tau_{F}=\omega_{F} \times \widehat{I} \omega_{F}+\widehat{I} \dot{\omega}_{F}
$$

The second of these three equations describes the flapping motion of the flybar. Although the complete expression is quite complex, it can be simplified by considering the pitch and roll motions of the helicopter independently. In addition, the small angle approximation is used for $\beta$ since it should not exceed $\pm 25^{\circ}$. For example, if roll motion only is considered, the second equation of (12) becomes

$$
\begin{gathered}
\ddot{\beta}+\cos \xi \ddot{\phi}-2 \Omega \sin \xi \dot{\phi}-\sin ^{2} \xi \beta \dot{\phi}^{2}+\Omega^{2} \beta= \\
\frac{1}{8 I_{f}} \rho \Omega^{2} a c\left(R_{2}^{4}-R_{1}^{4}\right)\left(\frac{\delta_{\phi}}{L_{5}} \sin \xi-\frac{\dot{\beta}}{\Omega}-\frac{\dot{\phi}}{\Omega} \cos \xi\right)
\end{gathered}
$$

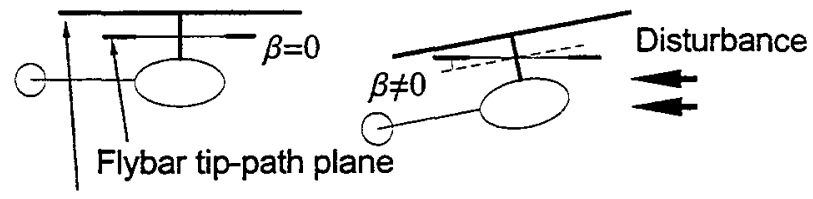

Main blade tip-path plane

Fig. 4 The stabilizing effect of the flybar. In a hovering situation, the flybar angle $\beta$ is zero. If a wind gust or other disturbance knocks the helicopter out of its equilibrium, the flybar, which is hinged freely, will continue to rotate in the same inertial plane. Its angle with respect to the main blade becomes nonzero, and it will help bring the helicopter back to equilibrium through its action on the cyclic angle of the main blade.

In the absence of aerodynamic forces and external moments, the flybar behaves as a gyroscope, maintaining its orientation relative to inertial space, ${ }^{16}$ as shown in Figure 4. An external disturbance would upset the helicopter angles $\theta$ and $\phi$, effectively changing $\beta$. This nonzero $\beta$ acts to apply an appropriate compensation input to the main blade cyclic control system to stabilize the helicopter.

\section{Actuator dynamics}

Once the flybar dynamics have been found, the rest of the actuator dynamics can be derived. The thrust generated by the rotor blade is $T$, and the moments represent the moments created by the rotor blade around the roll and the pitch axes are $M_{\phi}$ and $M_{\theta}$ respectively.

The moment created by a roll cyclic input is derived by summing the forces around a revolution and along a rotor blade. As per Figure 2, a positive pitch input $\delta_{\theta}$ produces roll moment $M_{\phi}$, but acts as pitch moment $M_{\theta}$ due to the precession effect. ${ }^{16}$

$$
M_{\phi}=\frac{1}{2 \pi} \int_{0}^{2 \pi} \int_{0}^{R} r \sin \xi d L_{m} d \xi
$$

We assume that the rotor angular velocity is constant; thus $\xi=\Omega t$. The lift element equation is similar to the flybar lift used in equation (10).

$$
d L_{m}=\frac{1}{2} \rho \Omega^{2} r^{2} a\left(\theta_{c y c}-\frac{v_{i}}{\Omega r}\right) c d r
$$

The induced air velocity $v_{i}$ must be derived empirically. ${ }^{16}$ However, it is usually of similar magnitude around the rotor disk, especially in hover or lowvelocity motions. It therefore has little effect on the net moment on the helicopter; we will assume that it is negligible in calculating $d L_{m}$.

From Figure 5, there are two different kinds of inputs which affect $\theta_{\text {cyc }}$ : a direct input by the pilot and an indirect input by the flybar. The geometry 
of the hub linkages indicate that $\theta_{c y c}$ will be the weighted sum of these two inputs.

$$
\theta_{c y c}=\frac{L_{3}}{L_{1}\left(L_{2}+L_{3}\right)} \delta_{c y c}+\frac{L_{2} L_{4}}{L_{1}\left(L_{2}+L_{3}\right)} \beta
$$

The expression for the rotor thrust $T$ is wellknown in the literature. ${ }^{17}$

$T=\frac{1}{2} a \sigma \rho \pi R^{4} \Omega^{2}\left(\frac{B^{3}}{3} \theta_{\circ}-\frac{B^{2}}{2} \lambda+\frac{B^{2}}{2} \mu \frac{1}{L_{1}} \sqrt{\delta_{\phi}^{2}+\delta_{\theta}^{2}}\right)$

The constant tip loss factor $B$ takes into account the fact that a finite length rotor blade would lose some of the lift generated due to the wing tip vortex effect; ${ }^{16}$ we will use the value $B=0.97 .{ }^{17}$ The throttle stick position $\delta_{t}$ also influences the collective pitch angle $\theta_{0}$ through a simple smooth function, $K_{\theta_{0}}$. Under normal operating conditions, these two effects balance each other, and $\Omega$ is expected to remain constant. ${ }^{16}$ Doing so also ensures constant cyclic control gain. Recall that $\theta_{0}$ is the collective pitch angle, and $\delta_{\phi}$ and $\delta_{\theta}$ are the roll and pitch inputs respectively.

The yaw command $\delta_{\psi}$ directly influences the collective pitch of the tail rotor blades $\theta_{o T}$, and the throttle input $\delta_{t}$ is directly coupled both to the motor torque $T_{m}$ and the collective pitch of the main rotor blades $\theta_{0}$. We model these relationships as linear because their dynamics are fast compared to the main rotor dynamics.

$$
\begin{aligned}
\theta_{o T} & =K_{\theta_{\circ T}} \cdot \delta_{\psi} \\
\theta_{0} & =K_{\theta_{0}} \cdot \delta_{t} \\
T_{m} & =K_{T_{m}} \cdot \delta_{t}
\end{aligned}
$$

The angular velocity of the tail rotor blades is related to the angular velocity of the main rotor blades through a constant $K_{\Omega}$.

$$
\Omega_{T}=K_{\Omega} \cdot \Omega
$$

The yaw moment $M_{\psi}$ is equal to the thrust of the tail rotor multiplied by the distance $L_{T}$ between the main and tail rotor axes. There is no cyclic input for the tail rotor blades; only a collective pitch angle. Thus, the yaw moment equation is similar to the thrust equation of the main rotor blade (15), replacing the terms with the tail rotor equivalents where appropriate.

$$
M_{\psi}=\frac{1}{2} a_{T} \sigma_{T} \rho \pi R_{T}^{4} \Omega_{T}^{2}\left(\frac{B^{3}}{3} \theta_{o T}-\frac{B^{2}}{2} \lambda_{T}\right) L_{T}
$$

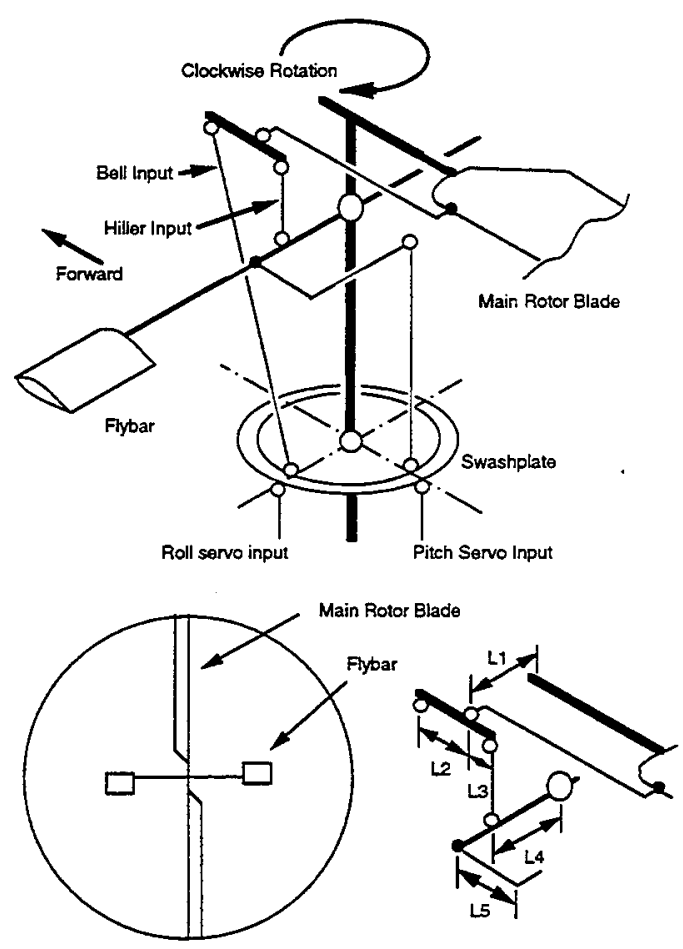

Fig. 5 The basic structure of the model helicopter's cyclic control system. The flapping angle of the flybar, $\beta$, is the angle of the flybar with respect to the body coordinate frame attached to the rotor hub. It is zero when the fiybar is perpendicular to the rotor axis. Ball joints are shown as "o", and fixed joints are shown as "๑". The cyclic pitch input to the main rotor blade is controlled by the combination of the Bell input from the swashplate and the Hiller input from the flybar.

The subscripts $T$ indicate that the values pertain to the tail rotor. To calculate the inflow ratio of the tail rotor $\lambda_{T}$, we can use equation(6). First, equation(2) is used to compute the velocity of the tail rotor hub in body coordinates; $q_{b}$ must be replaced $\left[\begin{array}{lll}-L_{T} & 0 & 0\end{array}\right]^{T}$ which is the coordinate of tail rotor hub. Equation (5) can then be used to obtain $\nu_{T}$. Again, the induced air velocity $v_{i}$ must be estimated or obtained empirically.

\section{System Identification}

The system identification is performed by isolating the effects of each input, and considering only a single output. As a first step, we are attempting to identify some of the physical parameters of the helicopter while restricting to either roll or pitch motion only. The direct least squares method is applied to identify the coefficients of the linearized 


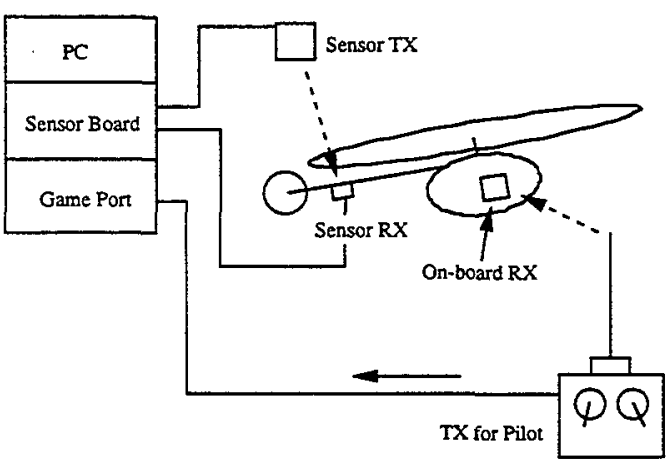

Fig. 6 A sketch of the experimental setup for the system identification of the helicopter's physical parameters. The helicopter is controlled through the radio transmitter by a human pilot. The computer is used to record the input data from the transmitter and the output data from the sensor.

SISO transfer function. The input and output data are collected while a pilot gives either a roll or pitch control input (the other inputs are held to zero).

For example, consider the roll motion of the helicopter. Putting together the roll equation from (1) and the flybar equation (13), it can be seen that the equation relating the roll input $\delta_{\phi}$ to the roll angle $\phi$ will be fourth order. The unknown parameters in the equation are the inertias $I_{x x}$ and $I_{f}$ and the lift curve slope $a$; the rotor angular velocity $\Omega$ can be measured directly. Because the input from the transmitter is held constant over each sample time, a discrete-time transfer function $H_{d}(z)$ can be used to represent the linearized system:

$$
H_{d}(z)=\frac{b_{1} z^{4}+b_{2} z^{3}+b_{3} z^{2}+b_{4} z+b_{5}}{z^{4}+a_{1} z^{3}+a_{2} z^{2}+a_{3} z+a_{4}}
$$

The coefficients $a_{i}, b_{i}$ will be identified using a least squares method; finding the best match between a linear SISO transfer function and the measured input-output data.

\section{Experimental setup}

A Polhemus sensor ${ }^{3}$ is used to measure the position and orientation of the helicopter; full six degreeof-freedom information $(x, y, z, \phi, \theta, \psi)$ is available at $50 \mathrm{~Hz}$. As shown in Figure 6, the sensor consists of a board connected to the PC's ISA slot, a transmitter, and a receiver. The transmitter is fixed to the ceiling, and it sends out a magnetic field via three orthogonal inductors. The receiver, fixed to the helicopter, senses the strength and the orientation of the magnetic field and sends this information back to the PC. For the preliminary experiments described here, we have fixed the helicopter to a stand to be able to concentrate on the pitch and roll motion. The input

\begin{tabular}{|l|c|}
\hline experiment & mean-squared error \\
\hline pitch SISO & 0.0002 \\
roll SISO & 0.0010 \\
pitch coupled & 0.0013 \\
roll coupled & 0.0014 \\
\hline
\end{tabular}

Table 1 A sampling of the mean-squared errors between the actual and simulated outputs using the identified transfer functions $H_{\delta_{\phi d}}(z)$ and $H_{\delta_{\theta d}}(z)$. Although the errors are slightly larger in the coupled experiment, they are still small enough to give some degree of confidence in the identified transfer function.

and output data are taken at $50 \mathrm{~Hz}$, for a total of approximately 2 minutes duration.

\section{$\underline{\text { Results }}$}

Once the input-output data has been taken and stored for the isolated roll motion, the system identification algorithm is run to estimate the discretetime transfer function coefficients $a_{i}$ and $b_{i}$, and thus the SISO transfer function $H_{\phi}(z)$. The initial condition of the system is also estimated using a least-squares method. The input data and the estimated transfer function are used to simulate the output data, and the simulated and actual output are compared. A reasonable match is achieved, as shown in Figure 7. The mean-squared error between the actual output $\phi_{a}$ and the simulated output $\phi_{s}$ from the estimated transfer function is computed as follows:

$$
\text { error }^{2}=\frac{1}{N} \sum_{i=1}^{N}\left(\phi_{a}(i)-\phi_{s}(i)\right)^{2}
$$

Typical values for this error on the order of $10^{-3}$ to $10^{-4}$.

A similar identification is performed for the pitch motion, and the SISO transfer function $H_{\theta}(z)$ is identified. The input data and the estimated transfer function are used to simulate the output data, and the simulated and actual output are compared. Again, a reasonable match is achieved, as shown in Figure 8.

To determine the validity of the single-axis identification, an experiment was performed in which both pitch and roll motions were commanded by the pilot. The input-output data was collected, and the input data was used with the single-input transfer functions to simulate both the pitch and roll outputs. The results from this experiment are given in Figure 9. There is some coupling between the pitch and roll motions, but it does not overwhelm the dominant single-input effects. A sampling of the mean-squared errors is given in Table 1. 


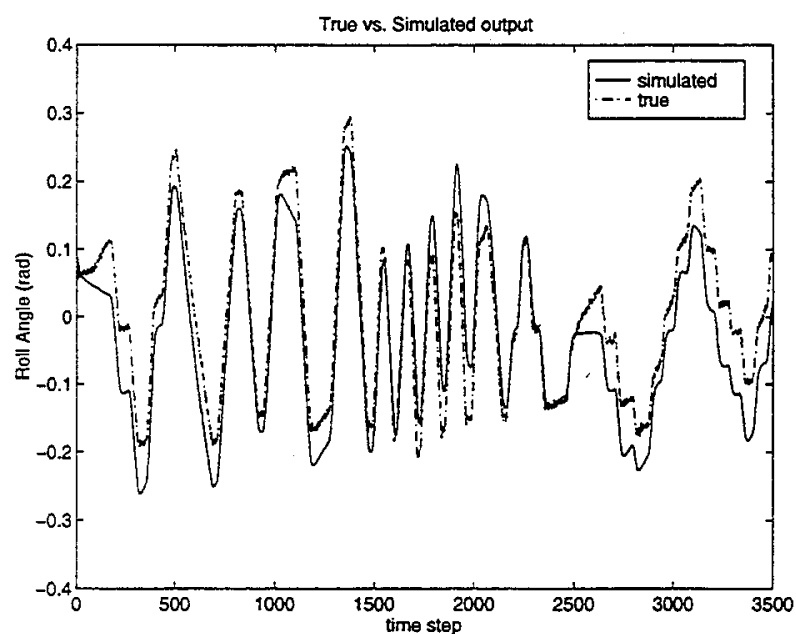

Fig. 7 The comparison between the simulated roll output based on the identified discrete time transfer function and the actual output from the experiment, given the identical roll input command history. The initial value for the simulation was also determined with least squares method with first $\mathbf{5 0}$ points from the experiment.

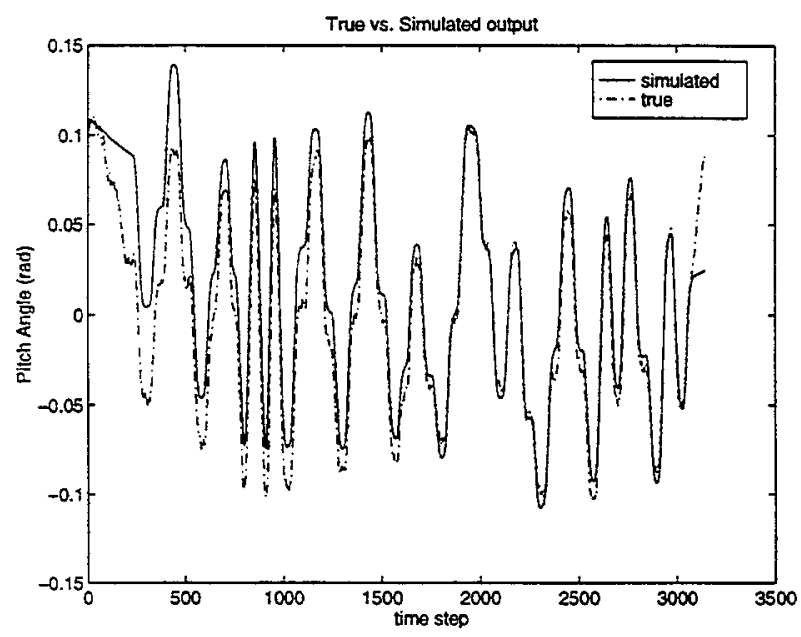

Fig. 8 The comparison between the simulated pitch output based on the identified discrete time transfer function and the actual output from the experiment, given the identical pitch input command history. The initial value for the simulation was also determined with least squares method using the first $\mathbf{5 0}$ points from the experiment.
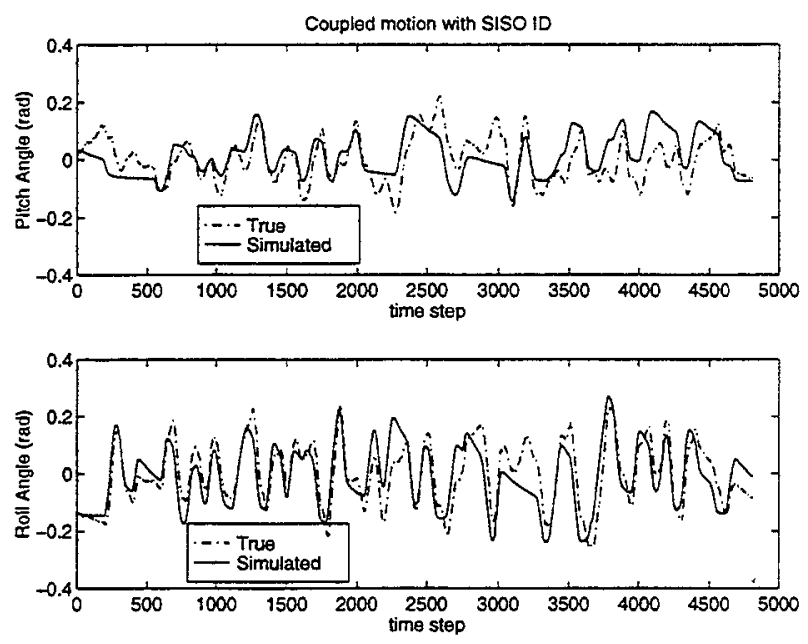

Fig. 9 The system identification result is applied to the pitch and roll motion simultaneously. The figure shows some coupled responses through the discrepancy between the true and the simulated result.

\section{Conclusions and Future Work}

A model helicopter is significantly different than a full-scale helicopter-it is faster in response, and lacks stability. A stability augmentation device called a flybar is commonly used to help the pilot control the model helicopter. The main contribution of this paper is to model the interaction between this flybar and the main rotor blade assembly, and to use these dynamic equations to derive a new and complete mathematical model for the dynamics of a model helicopter. Some preliminary system identification experiments were also presented.

We plan to continue the system identification, and use the results to identify the some of the physical parameters of the helicopter such as the inertias, aerodynamic coefficients, and other constants (such as the gains $K_{\theta_{0}}$, etc.). These parameters will be used to get an accurate nonlinear model for the helicopter dynamics using the equations outlined in the modeling section of this paper. Further experiments will then be performed to validate the nonlinear model. Once we have a fairly accurate nonlinear model, we will begin our feedback control experiments. A short-term goal of this project is to autonomously hover the helicopter in the lab. A longer-term goal is to use the complete nonlinear model to study the interaction between path planning and feedback control for autonomous vehicles, including the model-scale helicopter. 


\section{Nomenclature}

a main rotor lift slope

$a_{T} \quad$ tail rotor lift slope

$B$ tip loss factor

C main rotor blade chord length

$D_{F_{x, y}, z} \quad$ fuselage profile drag forces

$d L, d L_{m} \quad$ differential lift elements for flybar

and main rotor blade

$g$ gravitational acceleration

$h_{r} \quad$ distance between rotor disk and CG, parallel to rotor axis

$\hat{I} \quad$ rotational inertia matrix of flybar

$I_{3 x 3} \quad 3 \times 3$ identity matrix

$I_{f} \quad$ flybar moment of inertia in flapping

$I_{\tau} \quad$ rotor moment of inertia around rotor axis

$I_{x x, y y, z z, x z}$ fuselage rotational moments of inertia

$\mathcal{I}$ rotational inertia matrix of helicopter

$K_{g} \quad$ gyro gain for tail rotor

$K_{T_{m}} \quad$ proportional constant relating $\delta_{t}$ to $T_{m}$

$K_{\theta_{0}} \quad$ proportional constant relating $\delta_{t}$ to $\theta_{0}$

$K_{\theta_{\circ} T} \quad$ proportional constant relating $\delta_{t}$ to $\theta_{o T}$

$K_{\Omega} \quad$ proportional constant relating $\Omega$ to $\Omega_{T}$

$\ell_{r} \quad$ distance between rotor axis and CG, perpendicular to rotor axis

$L_{T} \quad$ distance between tail rotor axis and CG

$L_{1} \ldots L_{5} \quad$ linkage lengths in rotor hub assembly

$m \quad$ helicopter total mass

$m_{F} \quad$ Flybar mass per length

$M_{\phi, \theta, \psi} \quad$ net moments on helicopter

$R \quad$ length of main rotor blade

$R_{T} \quad$ length of tail rotor blade

$R_{1} \quad$ distance between rotor axis and flybar tip

$R_{2} \quad$ distance between rotor axis and flybar root

$R_{I B, B F, Y F}$ rotation matrices between Inertial, Body, and Flybar frames

$T \quad$ net thrust generated by rotor

$T_{m} \quad$ torque applied by motor

$v_{i} \quad$ induced air velocity through rotor disk

$v_{I B}^{b} \quad$ linear velocity of helicopter

$x, y, z \quad$ helicopter position coordinates

$\beta \quad$ flybar flapping angle

$\delta_{c y c} \quad$ cyclic input displacement

$\delta_{f t y} \quad$ cyclic input to flybar

$\delta_{\theta}, \delta_{\phi}, \delta_{\psi} \quad$ roll, pitch, and yaw command input

$\delta_{t}$

$\theta_{A O A}$

$\theta_{0}$

$\theta_{O T}$

$\hat{\theta}_{\text {cyc }}$

$\lambda$

$\lambda_{T}$

$\lambda_{T}$

$\mu$

$\nu_{T}$

$\rho$

$\sigma_{T}$

$\tau_{F}$ $\phi, \theta, \psi \quad$ helicopter angular position

$\omega_{I B}^{b} \quad$ angular velocity of helicopter

$\omega_{F} \quad$ angular velocity of flybar

$\Omega \quad$ main rotor angular velocity

$\Omega_{T} \quad$ tail rotor angular velocity

\section{References}

${ }^{1}$ Morris, J., Nieuwstadt, M., and Bendotti, P., "Identification and Control of a Model Helicopter in Hover," Proceedings of the American Control Conference, Vol. 2, 1994, pp. 12381242.

${ }^{2}$ Zhu, X. and Nieurstadt, M., "The Caltech Helicopter Control Experiment," CDS Technical Report 96-009, 1996.

${ }^{3}$ Polhemus Incorporated, P.O. Box 560, Colchester, VI 05446, 3SPACE User's Manual, Dec. 1993.

${ }^{4}$ Sugeno, M., "Fuzzy hierarchical control" of an unmanned helicopter," 1994, available at ftp://ftp.cs.arizona.edu/japan/kahaner.reports/sugeno.94.

${ }^{5}$ Fagg, A., Lewis, M., Montgomery, J., and Bekey, G., "The USC autonomous flying vehicle: An experiment in realtime behavior-based control," Proceedings of the IEEE Intl Conference on Intelligent Robots and Systems, Vol. 2, 1993, pp. $422-429$.

${ }^{6}$ Montgomery, J., Fagg, A., and Bekey, G., "AFV-I: A behavior-based entry in the 1994 international aerial robotics competition," IEEE Expert, Vol. 10, No. 2, Apr. 1994, pp. 1622.

${ }^{7}$ Johnson, E., DeBitetto, P., Trott, C., and Bosse, M., "The $1996 \mathrm{MIT} /$ Boston Univ/Draper Lab Autonomous Helicopter System," Proceedings of the 15th AIAA/IEEE Digital Avionics Systems Conference, 1996, pp. 381-386.

${ }^{8}$ Ozimina, C., Tayman, S., and Chaplin, H., "Flight Control System Design For A Small Unmanned Aircraft," Proceedings of the American Control Conference, Vol. 5, Jun. 1995, pp. 2964-2969.

${ }^{9}$ Kaminer, I., Hallberg, E., Pascoal, A., and Silvestre, C., "On the Design and Implementation of a Trajectory Tracking Controller for a Fixed Wing UAV," Proceedings of the American Control Conference, Jun. 1995, pp. 2970-2974.

${ }^{10}$ Thornberg, C. and Cycon, J., "Sikorsky Aircraft's UAV, Cypher: System Description and Program Accomplishments," Proceedings of the American Helicopter Society 51 st Annual Forum, May 1995.

${ }^{11}$ Pallett, T., Real-time Helicoper Flight Control, Master's thesis, School of Electrical Engineering, Purdue University, Aug. 1991.

${ }^{12}$ Furuta, K., Ohyama, Y., and Yamano, O., "Dynamics of RC helicopter and Control," Mathematics and Computers in Simulation XXVI, 1984, pp. 148-159.

${ }^{13}$ Azuma, A., "Dynamic Analysis of the Rigid Rotor System," Journal of Aircraft, Vol. 4, No. 3, 1967.

${ }^{14}$ Padfield, G., Helicopter Flight Dynamics, Blackwell Science Ltd, 1996.

${ }^{15}$ Murray, R., Li, Z., and Sastry, S., A Mathematical Introduction to Robotic Manipulation, CRC Press, 1994.

${ }^{16}$ Johnson, W., Helicopter Theory, Dover Publications, Inc., 1980.

${ }^{17}$ Okuno, Y., Kawachi, K., Azuma, A., and Saito, S., "Analytical Prediction of Height-Velocity Diagram of a Helicopter Using Optimal Control Theory," Journal of Guidance, Control, and Dynamics, Vol. 14, No. 2, 1991, pp. 453-459.

${ }^{18}$ Hostetler, R., RAY's complete helicopter manual, R/C modeler corporation, 1991.

${ }^{19}$ Astrom, K. and Wittenmark, B., Adaptive Control, Addison-Wesley Publishing Company, 1995. 